\title{
Zeeman coherence and quantum beats in ultrafast photon echoes of $\mathrm{N}-\mathrm{V}$ centers in diamond
}

\author{
S.C. Rand*, A. Lenef, S.W. Brown \\ Division of Applied Physics, 1049 Randall Laboratory, University of Michigan, Ann Arbor, MI 48109-1120, USA
}

\begin{abstract}
Ultrafast photon echo experiments reveal highly modulated quantum beats in nitrogen-vacancy centers in diamond, providing direct measurements of excited state splittings for the first time. We also confirm the existence of new peaks in echo excitation spectra and report novel polarization dependent interferences.
\end{abstract}

The establishment of definitive models of luminescent centers in solids, particularly in widegap semiconductors, is becoming increasingly important in the context of next generation flat panel displays. Here, we report the direct determination of excited state structure and dephasing dynamics of the nitrogen-vacancy $(\mathrm{N}-\mathrm{V})$ center in diamond from ultrafast photon echo observations. These results provide the basis for a detailed model of the center.

Previous results from isochronal annealing experiments and uniaxial stress measurements by Davies and Hamer [1] on the $638 \mathrm{~nm}$ zero phonon transition of the N-V center provided evidence for a trigonal center of $\mathrm{C}_{3 v}$ symmetry consisting of a carbon vacancy adjacent to a nitrogen impurity. The ground and excited states were determined to be of A and E symmetry, respectively [1]. Electron paramagnetic resonance (EPR) measurements by Loubser and Van Wyk [2] confirmed the basic physical model of the center. Transient hole-

\footnotetext{
* Corresponding author.
}

burning experiments [3] and Curie-law dependence of the magnetic susceptibility from ground state EPR measurements at $2.88 \mathrm{GHz}$ by Redman et al. [4] established the triplet character of the ground state. Persistent hole-burning measurements also revealed transitions at frequencies combining the ground state spin-spin splitting with other frequencies on the $\mathrm{GHz}$ scale, providing indirect evidence for ${ }^{3} \mathrm{E}$ excited state structure [5]. Optically detected microwave resonance (ODMR) measurements [6], in which the laser probe was tuned across the ZPL, also revealed a larger $40 \mathrm{~cm}^{-1}$ splitting in the ODMR spectrum, suggesting that an even larger splitting existed in the excited state.

In the present work, ultrafast stimulated photon echo experiments were performed to investigate excited state structure directly. A dual jet, synchronously-pump DCM dye laser was employed, generating cavity-dumped subpicosecond pulses at a variable repetition rate near $100 \mathrm{KHz}$. The sample was a high-quality synthetic, nitrogen rich single crystal. This was irradiated with $1.7 \mathrm{MeV}$ electrons and subsequently annealed to produce 

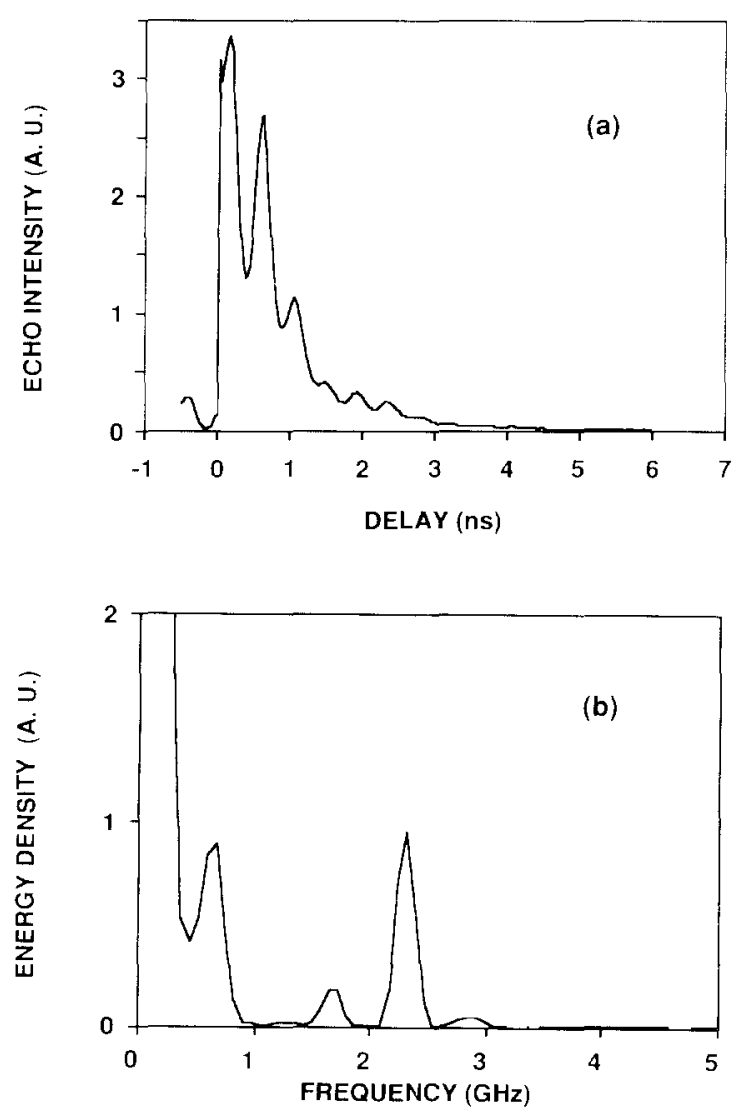

Fig. I. (a) Stimulated photon echo decay data from diamond $\mathrm{N} V$ centers, showing highly modulated quantum beats at $6 \mathrm{~K}$. $i=6.38 .2 \mathrm{~nm}$. Incident beams are all linearly polarized in the $001)$ direction. (b) Power spectrum of echo data.

high concentrations of $\mathrm{N}-\mathrm{V}$ centers. Three input beams were arranged in a phase conjugate geometry with counter-propagating pump waves and a probe wave incident at a small angle with respect to the forward pump. Pump waves were aligned parallel to the $[100]$ direction. Spatially filtered. time-averaged echo signals were recorded using lock-in detection techniques with a photomultiplier. Signals were recorded as a function of probe delay, incident wavelength, and temperature, and they verified to be independent of repetition rate. For polarization-dependent studies, quarter-wave plates were inserted into all three incident beams, using an additional quarter-wave plate and polarizer combination to analyze the resulting echo.
The photon echo intensity is shown in Fig. 1(a) as a function of probe delay, revealing deep modulations in the photon echo decay. The wavelength was tuned to the red side of the ZPL $(i=6.38 .2 \mathrm{~nm})$ with all incident beams polarized in the 001 , direction. Figure 1(b) shows the power spectrum of these data. The exponential decay in Fig. 1(a) was first divided from the data before Fourier transformation. We measured frequency components at $A=2.87 \pm 0.01 \mathrm{GH} x$, corresponding to the known ground state splitting. as well as components at $B=0.61 \pm 0.02 \mathrm{GH} z, \quad C=1.65 \pm 0.01 \mathrm{GH} \%$, and $D=2.30 \pm 0.01 \mathrm{GH} z$. related to excited state splittings. These observed frequencies agree with our persistent hole-burning observations [5]. but in addition confirm that all modulations originate directly from splittings in the ${ }^{3} \mathrm{~F}$ and ${ }^{3} \mathrm{~A}$ states coupled by the light. rather than relaxed states, since the signal appears as a quantum beat from coherence established within the incident pulse duration.

The existence of two ${ }^{3} \mathrm{E}$ excited states with different dephasing behaviors was revealed directly by recording the excitation spectrum of the echo signals across the ZPL. The excitation spectrum shows two distinct peaks separated by a width of $42 \pm 2 \mathrm{~cm}^{1}$; not resolvable in linear absorption measurements of these strongly inhomogeneously broadened centers. The dephasing rates versus temperature of these two states were measured with the laser tuned to the red $(638.9 \mathrm{~nm})$ and blue $(6.36 .5 \mathrm{~nm})$ sides of the ZPL linear absorption peak. Dephasing times at $5.5 \mathrm{~K}$ for the red and blue peaks were $4.92 \pm 0.24 \mathrm{~ns}$ and $56 \pm 8 \mathrm{ps}$, respectively, corresponding to four times the measured decay times in the photon echo signals. Theoretical fits confirm that the upper of the two ${ }^{3} \mathrm{E}$ states decays rapidly by a direct process to the lower level as one might expect, while the lower state dephases more slowly through a virtual 2 -phonon process. The dominant activation energy for the direct process was $67 \pm 25 \mathrm{~cm}$ '. in reasonable correspondence with the measured splitting of the two ${ }^{3}$ E states. For the lower level, an Orbach process dominates at low temperatures with an activation energy of $39 \pm$ $10 \mathrm{~cm}$ '. also in good agreement with the measured excited state splitting.

Finally, a detailed investigation of the polarization dependence of the echo intensities with circu- 
larly polarized beams revealed an interesting selection rule which caused signal intensities to drop by an order of magnitude when the pulse polarization sequence was $\sigma^{+} \sigma^{-} \sigma^{+}$or $\sigma^{-} \sigma^{+} \sigma^{-}$. This effect arises from interferences in the echo signal due to a superposition of third-order interactions in the presence of ground state magnetic degeneracies. For fixed probe delay, ignoring quantum beat contributions and population relaxation, the $i$ th component of the echo polarization $P_{i}^{(3)}$ has the form

$$
\begin{aligned}
P_{i}^{(3)}= & 2 \operatorname{Re}\left[\frac{1}{8} \sum_{x} \sum_{j k l} \varepsilon_{0} \chi_{i j k l}^{(3)}(x)\right. \\
& \left.\times\left(E_{1 j}^{*} E_{2 k} E_{3 l}+E_{1 j}^{*} E_{2 l} E_{3 k}\right)\right],
\end{aligned}
$$

where $E_{\eta j}$ is the $j$ th component of the applied electric field of the $\eta$ th pulse, and $\chi^{(3) i j k l}(\alpha)$ is a thirdorder susceptibility that includes the tensoral nature of the interaction. The summation over $\alpha$ in Eq. (1) is over the different defect orientations in the lattice.

If we consider the specific example of a $\sigma^{+} \sigma^{-} \sigma^{+}$ sequence for a specific orientation, the $x$ component of the third-order polarization in Eq. (1) is

$$
\begin{aligned}
P_{x}= & \varepsilon_{0} E_{0}\left|E_{0}\right|^{2}\left[\left(\chi_{x x x x}^{(3)}-\chi_{x x y y}^{(3)}-\chi_{x y x y}^{(3)}-\chi_{x y y x}^{(3)}\right)\right. \\
& \left.-\mathrm{i}\left(\chi_{x y y y}^{(3)}-\chi_{x x x y}^{(3)}-\chi_{x x y x}^{(3)}-\chi_{x y x x}^{(3)}\right)\right]
\end{aligned}
$$

where $E_{0}$ is the magnitude of the applied fields. In a center with cubic symmetry, the only nonzero terms in Eq. (2) are $\chi_{x x y y}^{(3)}=\chi_{x y x y}^{(3)}=\chi_{x y y x}^{(3)}=\frac{1}{3} \chi_{x x x x}^{(3)}$, in which case $P_{x}=0$. For the more complicated case of the $\mathrm{C}_{3 \mathrm{v}}$ symmetry $\mathrm{N}-\mathrm{V}$ center, $P_{x}$ is still reduced significantly for the $\sigma^{+} \sigma^{-} \sigma^{+}$case when all defect orientations are considered. Explicit grouptheoretical calculations of $\chi^{(3)}$ for a transition between a doubly degenerate $\mathrm{E}$ symmetry state in the ground state manifold and a nondegenerate excited state in the $\mathrm{N}-\mathrm{V}$ center predict relative echo intensities with ratios of $64: 25: 4$ for $\sigma^{+} \sigma^{+} \sigma^{-}, \sigma^{+} \sigma^{+} \sigma^{+}$ or $\sigma^{+} \sigma^{-} \sigma^{-}$, and $\sigma^{+} \sigma^{-} \sigma^{+}$polarization sequences (and equivalent sequences with reversed circular polarization), respectively. These results, which will be presented in detail in a later work, assume applied fields propagate in the $\left[\begin{array}{lll}0 & 0 & 1\end{array}\right]$ direction and are in good agreement with our observations.

In summary, we have presented quantum beat measurements of excited state splittings in the N-V center in diamond. The existence of a large splitting in the excited state manifold was revealed in echo excitation spectra. Temperature-dependent dephasing studies of these two states indicated that direct and virtual 2-phonon processes were the primary mechanisms responsible for dephasing from the upper and lower ${ }^{3} \mathrm{E}$ levels, respectively. Finally, we observed a polarization selection rule which can be explained as an interference effect due to Zeeman coherence within the center which is consistent with our model of the electronic structure.

\section{Acknowledgements}

This research was sponsored in part by the Air Force Office of Scientific Research and the National Science Foundation and Technology Center for Ultrafast Optical Science (STC PHY 8920108). A. Lenef gratefully acknowledges support from the Department of Education in the form of a DSO fellowship.

\section{References}

[1] G. Davies and M.F. Hamer, Proc. Roy. Soc. London A 348 (1970) 285

[2] J.H.N. Loubser and J.A. Van Wyk, Diamond Research (Industrial Diamond Information Bureau, London, 1977) p. 11.

[3] N.R.S. Reddy, N.B. Manson and E.R. Krausz, J. Lumin. 38 (1987) 46.

[4] D.A. Redman, S.W. Brown, R.H. Sands and S.C. Rand, Phys. Rev. Lett. 67 (1991) 3420.

[5] D.A. Redman, S.W. Brown and S.C. Rand, J. Opt. Soc. Am. B 9 (1992) 768.

[6] E. van Oort, B. van der Kamp, R. Sitters and M. Glasbeek. J. Lumin. $48 \& 49$ (1991) 803. 